\title{
Diffusion-weighted MRI Provides a Useful Biomarker for Evaluation of Radiotherapy Efficacy in Patients with Prostate Cancer
}

\author{
XINGCHEN WU ${ }^{1,2,3}$, PETRI REINIKAINEN ${ }^{1}$, MIKA KAPANEN ${ }^{1,4}$, TUULA VIERIKKO ${ }^{3}$, \\ PERTTI RYYMIN ${ }^{3,4}$ and PIRKKO-LIISA KELLOKUMPU-LEHTINEN ${ }^{1,2}$ \\ ${ }^{1}$ Department of Oncology, ${ }^{3}$ Department of Radiology and ${ }^{4}$ Department of Medical Physics, \\ Medical Imaging Centre, Tampere University Hospital, Tampere, Finland; \\ ${ }^{2}$ Faculty of Medicine and Life Sciences, University of Tampere, Tampere, Finland
}

\begin{abstract}
Background: Diffusion-weighted imaging (DWI) with measurement of apparent diffusion coefficient (ADC) allows for assessment of tumor aggressiveness. The objective of this study was to evaluate the changes of $A D C$ value in prostate cancer after volumetric-modulated arc radiotherapy (VMAT) and to identify magnetic resonance imaging (MRI) biomarkers for monitoring tissue changes after radiotherapy. Patients and Methods: Thirty-seven patients with biopsyproven prostate cancer treated with VMAT underwent serial MRI examinations including DWI before radiotherapy, and at 3 and 12 months after radiotherapy. ADC values of the tumor and healthy prostate tissue were measured and compared at these three time points. Results: The tumor ADC value increased significantly 3 months after radiotherapy $(p<0.0001)$. There was a further increase of tumor $A D C$ from 3 to 12 months after radiotherapy $(p<0.01)$. The ADC of healthy prostate tissue did not show any significant changes. Conclusion: The ADC value is a useful biomarker for evaluating the efficacy of radiotherapy in prostate cancer.
\end{abstract}

External-beam radiotherapy is considered a standard treatment option for patients with localized prostate cancer (1). Determination of the serum prostate-specific antigen (PSA) level is currently the most widely used test for monitoring the response to radiotherapy (2). However, nearly one-third of

This article is freely accessible online.

Correspondence to: Xingchen Wu, Senior Researcher, MD, Ph.D., Department of Oncology, Tampere University Hospital, Tampere, Finland. Tel: +358 405314642, Fax: +358 331163009, e-mail: Xingchen.Wu@uta.fi

Key Words: Prostate cancer, diffusion-weighted MRI, ADC, Gleason score, radiotherapy, hypofractionation. patients will experience at least one PSA 'bounce' after radiotherapy and about half of the patients with a postradiotherapy PSA bounce will not develop cancer recurrence (3), indicating that a rising PSA level is not a reliable indicator of tumor recurrence, even though a lower PSA nadir after radiotherapy has been associated with cancer cure (4). Furthermore, no pattern of PSA kinetics after radiotherapy has conclusively differentiated between local and distant failure, which is a clinical challenge (4-6). In this setting, medical imaging plays an important role in differentiating local recurrence from distant metastatic disease. Imaging techniques are useful in detecting distant metastases, such as those in the bone, and in assessing lymph node invasion (7). However, precise delineation of the location and extent of recurrent tumor within the prostate after radiotherapy continues to be difficult with all imaging techniques $(7,8)$.

Magnetic resonance imaging (MRI) has been used to assess local tumor recurrence after radiotherapy. However, T2weighted imaging has shown limited accuracy for tumor detection and localization in prostate cancer (9). In addition, tissue changes such as glandular atrophy and fibrosis induced by radiation cause a decrease in T2-weighted signal intensity within the prostate and make cancer lesions less conspicuous compared to benign prostatic tissue $(7,10)$, which limits the use of conventional T2-weighted imaging in monitoring efficacy after radiotherapy. Diffusion-weighted imaging (DWI) is a noninvasive, functional MR technique that allows quantification of Brownian motion of water molecules in tissue, thereby enabling assessment of tumor aggressiveness $(11,12)$. DWI with measurement of the apparent diffusion coefficient (ADC) is promising in improving tumor detection and characterization $(11,12)$. Previous studies have shown that DWI reflects cellular changes in malignant tissue under radiotherapy and that the DWI ADC is a potential tool for the assessment of treatment efficacy in prostate cancer during and shortly after radiotherapy (13-16). However, well-defined prospective long-term 
observations are needed, especially for comparing different radiotherapy regimens. Recent data suggest that prostate cancer might be sensitive to radiation fraction size, which would give a therapeutic advantage to hypofractionated treatment $(17,18)$; these findings warrant confirmation.

This study was designed to evaluate changes of ADC in biopsy-proven prostate cancer treated with either conventional or moderate hypofractionated radiotherapy regimen, and to identify and quantify suitable MRI parameters for monitoring tissue changes after radiotherapy.

\section{Patients and Methods}

Patients. Forty-four consecutive patients with histologically proven prostate adenocarcinoma were enrolled in our prospective clinical trial (NCT 02319239). The inclusion and exclusion criteria were described in detail in our previous publication (11). Briefly, we included adult patients with one or two intermediate-risk features (Gleason score 7, staging T2b-T2c, PSA 10-20 ng/ml) according to the National Comprehensive Cancer Network Clinical Practice Guidelines in Oncology NCCN Guidelines ${ }^{\circledR}$ (19), without antiprostate cancer hormonal therapy history, and who were suitable for MRI examination. The study was approved by the local Ethics Committee (Nr. R14009), and all patients gave their written informed consent prior to study entry. All patients underwent physical examination, digital rectal examination, and standard laboratory tests including serum PSA. In addition, transrectal ultrasound-guided biopsy (12 cores, six on each lobe) was performed in each patient. Pathology was reviewed and graded according to the Gleason system. To minimize post-biopsy artifacts, MRI was performed 6-10 weeks after prostate cancer confirmation by biopsy.

Radiotherapy. The patients were allocated into two groups for radiotherapy: 18 patients were treated with conventional regimen external beam radiotherapy with 39 sessions of 2 Gy per fraction in 7.8 weeks ( $39 \times 2$ Gy) (group I), and 19 patients were treated with moderate hypofractionated regimen external beam radiotherapy: 20 sessions of 3 Gy per fraction in 4 weeks $(20 \times 3$ Gy) (group II). Radiotherapy was given with TrueBeam STx accelerator (Varian Medical Systems, Palo Alto, CA, USA) using volumetric-modulated arc therapy (VMAT) technique and two full arcs of $6 \mathrm{MV}$ flattened beams.

Time points of serial MRI and serum PSA determination. All patients were followed-up clinically throughout the study, and were imaged by multi-parametric MRI before radiotherapy initiation (E1), 3 months after radiotherapy completion (E2), and again 12 months after radiotherapy completion (E3). The serum PSA level was assessed on the day of each MRI scan. In addition, our patients will be continuously monitored by serial PSA and symptom questionnaires for at least 3 years after radiotherapy.

Multi-parametric MRI acquisition. Multi-parametric MRI was acquired using a 3-T MR System (Siemens Trio-Tim, Erlangen, Germany) with a combination of six channel body matrix coil and six elements of 24-channel spine matrix coil positioned around the pelvis to cover the prostate. The acquisition methods were described in detail in our previous publication (11). Briefly, tri-planar T2weighted turbo spin-echo images from below the prostatic apex to above the seminal vesicles were obtained. DWI was acquired with a single-shot echo-planar sequence on the axial plane using three $b$ values $\left(50,400\right.$, and $\left.800 \mathrm{~s} / \mathrm{mm}^{2}\right)$, and with the same orientation and location used to acquire axial T2-weighted images. In order to help to rule out false-positive findings (i.e. to identify post-biopsy hemorrhage), dynamic contrast-enhanced MRI was performed with axial T1-weighted 3D volumetric interpolated breath-hold examination sequence that covered the entire prostate in consecutive sections. The MRI parameters were described in detail in our previous publication (11). Briefly, DWI with echo time (TE), $77 \mathrm{~ms}$; repetition time (TR), $3800 \mathrm{~ms}$; field of view (FOV), 221×260 mm; flip angle (FA), $90^{\circ}$; acquisition matrix (ACQ), $102 \times 160 \mathrm{~mm}$; slice/gap, 3.6/0 mm. T2-weighted axial, sagittal, and coronal imaging with TE, $100 \mathrm{~ms}$; TR, 4000-5000 ms; FOV, 200×200 mm; FA, $90^{\circ}$; ACQ, $288 \times 320 \mathrm{~mm}$; slice/gap, $3.0 / 0.6 \mathrm{~mm}$.

MRI analysis. All MRIs were reviewed on a syngo Multimodality Workplace (Siemens Healthcare Oy, Erlangen, Germany). The method of ADC measurement was described in detail in our previous publication (11). Briefly, The ADC value of each identified tumor was measured directly on the parametric ADC maps. The slice of the ADC map containing the largest tumor extent was selected for analysis and a region of interest (ROI) was drawn in the center of the tumor excluding the tumor edges. The mean ADC value of the tumor was generated at the workstation and recorded for analysis. Healthy control prostatic tissue was selected from the contralateral side of the tumor.

Follow-up MRI examination and analysis. The same MRI scanner was used throughout the study, and the same workstation was used for image analysis. In MRI follow-up examinations, the same anatomic locations and the same pack of imaging sequences were used. For the follow-up image analysis, in order to determine the ADC changes after radiotherapy, the same tumor location was analyzed for comparison and as an indicator of disease status. Anatomical landmarks on T2-weighted images and the respective lesions were used to locate the tumors on the follow-up images. An example of the tumor changes after radiotherapy in a series of MRIs is shown in Figure 1.

Statistical analysis. Statistical analysis was performed with SPSS (version 23.0; IBM Corp., Armonk, NY, USA). A two-sided nonparametric Mann-Whitney $U$-test was used to compare the ADC values between tumor and non-tumor prostatic tissue and the percentage change of ADC between the two radiotherapy regimen groups; paired $t$-test was used to compare the tumor ADC values and serum PSA before and after radiotherapy. Spearman's correlation coefficient was used to evaluate the correlation between the tumor ADC, Gleason score, PSA, and the changes of tumor ADC and PSA after radiotherapy, $p$-values of less than 0.01 were considered significant.

\section{Results}

Patient characteristics. No suspicious lesion was found on MRI in seven out of the 44 patients with prostate cancer, 43 lesions were detected in the remaining 37 patients (mean age $\pm \mathrm{SD}=69 \pm 5$ years, range $=60-79$ years $)$. There were 14 patients with Gleason score $3+4$, and 23 with Gleason score $3+3$. All 37 patients with detectable lesions had tumor in 


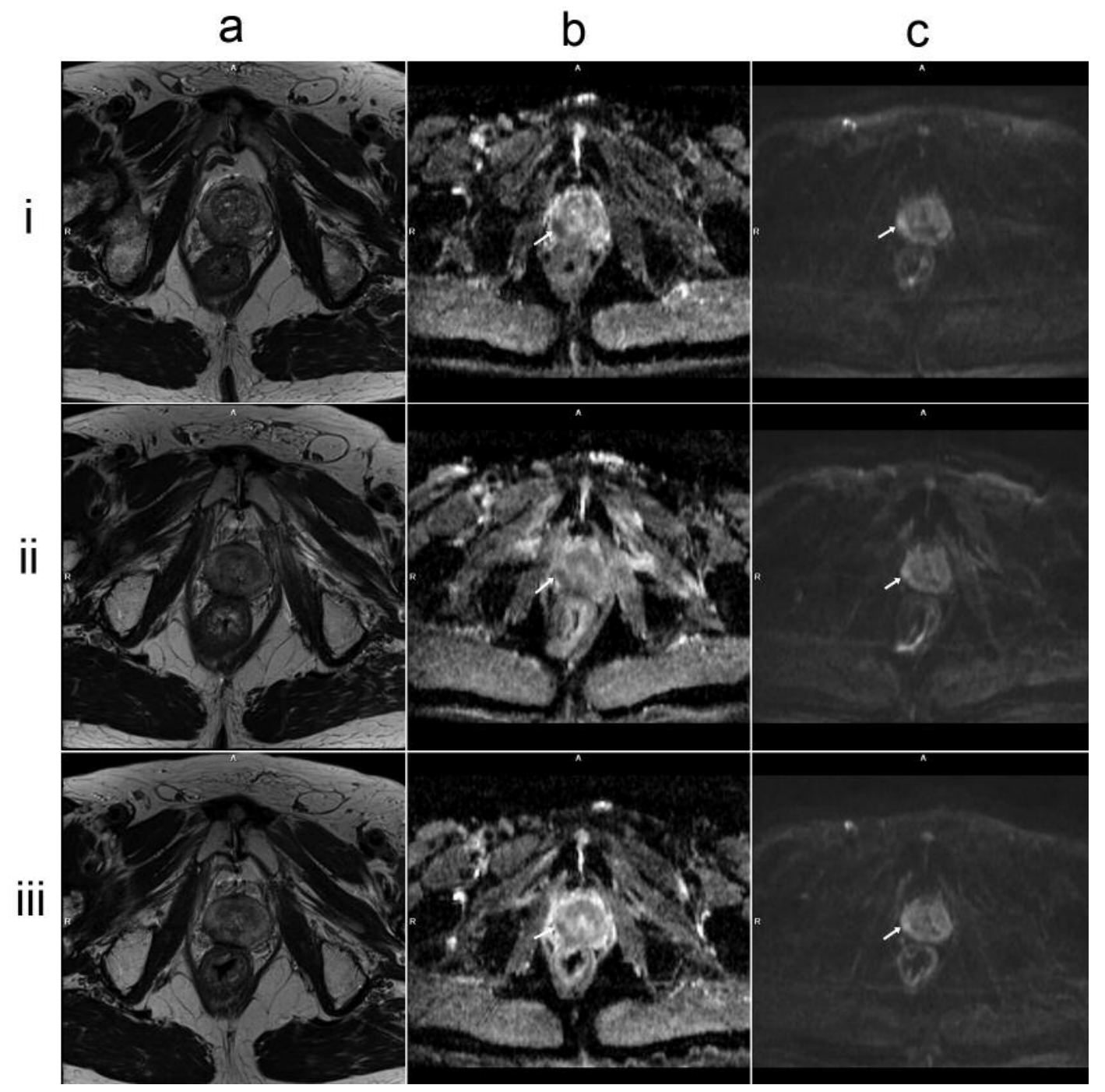

Figure 1. Prostate magnetic resonance imaging (MRI) from a 70-year-old man with biopsy-proven prostate cancer, Gleason score $3+4$ and serum prostate-specific antigen (PSA) $6.8 \mathrm{ng} / \mathrm{ml}$. The patient received $20 \times 3$ Gy radiotherapy after baseline MRI examination. The top row shows the axial prostate MRIs at baseline, the middle row shows those at 3 months after radiotherapy, and the bottom row shows those at 12 months after radiotherapy. a: T2-Weighted images: At baseline there was diffuse low signal intensity in the right peripheral zone (i); after radiotherapy (ii, iii) the pathological signal gradually disappeared. $b$ : Apparent diffusion coefficient (ADC) maps showing a clear lesion in the right peripheral region (arrows): at baseline (i) the tumor ADC value was $0.75 \times 10^{-3} \mathrm{~mm}^{2} / \mathrm{s}$; at 3 months after radiotherapy, the lesion size had decreased and the ADC value had increased to $1.14 \times 10^{-3} \mathrm{~mm}^{2} / \mathrm{s}$ (ii); at 12 months after radiotherapy, the lesion size had decreased further and the ADC value had increased to $1.39 \times 10^{-3} \mathrm{~mm}^{2} / \mathrm{s}$. c: Diffusion-weighted images, with b-value of $800 \mathrm{~s} / \mathrm{mm}^{2}$, showing the hyperintense lesion (arrows) corresponding to the lesion on ADC maps.

clinical stage T2a or T2b. Group I (18 patients with 23 lesions) received conventional regimen radiotherapy of $39 \times 2$ Gy: a fraction dose of 2 Gy in a single irradiation for five consecutive days in each week, lasting 7.8 weeks with a total prescription dose of 78 Gy; group II (19 patients with 20 lesions) received moderate hypofractionated regimen radiotherapy of $20 \times 3 \mathrm{~Gy}$ : a fraction dose of $3 \mathrm{~Gy}$ in a single irradiation for five consecutive days in each week, lasting 4 weeks with a total prescription dose of $60 \mathrm{~Gy}$. The demographic characteristics, baseline serum PSA value, Gleason score, tumor zone of origin, and tumor ADC value of the two groups of patients are provided in Table I.

Serum PSA value. At 3 months after radiotherapy, the mean \pm SD serum PSA value decreased significantly (by $\sim 3$-fold, $p<0.0001)$ in the 37 patients with prostate cancer, and decreased further 12 months after radiotherapy (by $\sim 3$-fold, $p<0.0001$ ) (Table II). Only one patient in group II had a PSA bounce 3 
Table I. Demographic characteristics, baseline Gleason score, serum prostate-specific antigen (PSA) value, tumor zone of origin, and apparent diffusion coefficient $(A D C)$ value of the 37 patients with prostate cancer under two radiotherapy regimens.

\begin{tabular}{|c|c|c|c|}
\hline Characteristic & $\begin{array}{l}\text { Radiotherapy: } \\
39 \times 2 \text { Gy } \\
(\mathrm{N}=18)\end{array}$ & $\begin{array}{c}\text { Radiotherapy: } \\
20 \times 3 \text { Gy } \\
(\mathrm{N}=19)\end{array}$ & $\begin{array}{l}\text { Total } \\
(\mathrm{N}=37)\end{array}$ \\
\hline $\begin{array}{l}\text { Age: mean } \pm \text { SD (range), } \\
\text { years }\end{array}$ & $\begin{array}{c}67 \pm 4 \\
(60-73)\end{array}$ & $\begin{array}{c}71 \pm 6 \\
(61-79)\end{array}$ & $\begin{array}{l}69 \pm 5 \\
(60-79)\end{array}$ \\
\hline \multicolumn{4}{|l|}{ Gleason score, $\mathrm{n}$} \\
\hline $3+3$ & 6 & 8 & 14 \\
\hline $3+4$ & 12 & 11 & 23 \\
\hline \multicolumn{4}{|l|}{ Zone of tumor origin } \\
\hline Peripheral & 16 & 13 & 29 \\
\hline Transitional & 6 & 7 & 13 \\
\hline $\begin{array}{l}\text { Serum PSA: mean } \pm \text { SD } \\
\text { (range) }, \mathrm{ng} / \mathrm{ml}\end{array}$ & $\begin{array}{l}10.6 \pm 5.3 \\
(3.9-26.7)\end{array}$ & $\begin{array}{c}9.3 \pm 4.7 \\
(3.4-21.3)\end{array}$ & $\begin{array}{c}9.9 \pm 5.0 \\
(3.4-26.7)\end{array}$ \\
\hline $\begin{array}{l}\text { Tumor ADC: } \text { mean } \pm \mathrm{SD} \text {, } \\
\times 10^{-3} \mathrm{~mm}^{2} / \mathrm{s}\end{array}$ & $0.87 \pm 0.13$ & $0.82 \pm 0.10$ & $0.84 \pm 0.12$ \\
\hline
\end{tabular}

$\mathrm{N}$ : Number of patients; There was no significant difference in age, Gleason score, PSA, or ADC value between the two different radiotherapy regimen groups.

months after radiotherapy (from baseline 11.9 to $16.3 \mathrm{ng} / \mathrm{ml}$ ), but this had decreased to within the normal range $(2.3 \mathrm{ng} / \mathrm{ml})$ at 12 months after radiotherapy.

$A D C$ value at baseline. The tumor $\mathrm{ADC}$ value was lower than that of the control prostatic tissue in the 37 patients with prostate cancer $(p<0.0001)$ (Table III). The tumor ADC value was significantly lower than that of control prostatic tissue in both group I and group II ( $p<0.0001$, respectively) (Table III).

$A D C$ value after radiotherapy. All 37 patients completed the three MRI examinations. A significant increase of the tumor ADC value was detected 3 months after radiotherapy $(p<0.0001)$ in the 37 patients with prostate cancer, and the tumor ADC value had increased further at 12 months after radiotherapy $(p<0.0001)$ (Table III).

In group I, the tumor ADC value had increased by $34 \%$ at 3 months after radiotherapy $(p<0.0001)$ in the 18 patients with prostate cancer, and there was an $11 \%$ further increase at 12 months after radiotherapy $(p<0.01)$ (Table III).

In group II, the tumor ADC value had increased by $55 \%$ at 3 months after radiotherapy $(p<0.0001)$ in the 19 patients with prostate cancer, and increased by a further $9 \%$ at 12 months after radiotherapy $(p<0.01)$ (Table III).

The total increase of the tumor ADC value was $48 \%$ in group I and $67 \%$ in group II at 12 months after radiotherapy compared to baseline ( $p<0.0001$, respectively). The ADC value of healthy control prostate tissue did not show any significant changes
Table II. Paired comparison of serum prostate-specific antigen (PSA) $(\mathrm{ng} / \mathrm{ml})$ in the 37 patients with prostate cancer under two radiotherapy regimens.

\begin{tabular}{lcll}
\hline Timepoint & $\begin{array}{c}\text { Radiotherapy: } \\
39 \times 2 \mathrm{~Gy} \\
(\mathrm{~N}=18, \text { mean } \pm \mathrm{SD})\end{array}$ & $\begin{array}{c}\text { Radiotherapy: } \\
20 \times 3 \mathrm{~Gy}\end{array}$ & $\begin{array}{c}\text { Total } \\
(\mathrm{N}=19, \text { mean } \pm \mathrm{SD})\end{array}$ \\
$\begin{array}{lcll}\mathrm{mean} \pm \mathrm{SD}) \\
\text { Baseline }\end{array}$ & $10.6 \pm 5.3$ & $9.3 \pm 4.7$ & $9.9 \pm 5.0$ \\
3 Months after RT & $3.3 \pm 3.0^{* * *}$ & $2.7 \pm 3.5^{* * *}$ & $3.0 \pm 3.3^{* * *}$ \\
12 Months after RT & $1.4 \pm 1.5^{* *}$ & $0.9 \pm 0.7^{*}$ & $1.1 \pm 1.2^{* * *}$ \\
\hline
\end{tabular}

$\mathrm{N}$ : Number of patients; Significantly different at $* p<0.01, * * p<0.001$ and $* * * p<0.0001$ compared to previous time point in the same group.

after radiotherapy (Table III). The percentage increase of tumor $\mathrm{ADC}$ value in group II was greater than that in group I $(p<0.01)$ at 3 months after radiotherapy, but there was no significant difference in the ADC changes between the two groups at 12 months after radiotherapy.

Correlation. No correlation was detected between the tumor ADC value and the Gleason score, serum PSA, or the changes of ADC and PSA after radiotherapy.

\section{Discussion}

The ADC value is sensitive to the microscopic motion of water molecules and allows for noninvasive characterization of biological tissues based on their water diffusion properties. The degree of restriction of water diffusion in biological tissues is related to tissue cell density and the integrity of cell membranes. The degree of water diffusion is more restricted in tissues with a high cellular density because of the presence of numerous intact cell membranes and restricted water content in the extracellular space (20). Our study showed that the tumor ADC value was lower than that of the healthy control prostatic tissue in the 37 patients with prostate cancer.

Our study demonstrated that the ADC value of prostate cancer increased significantly 3 months after radiotherapy compared to that pre-therapy, and it increased further at 12 months after radiotherapy. These findings suggest that the ADC value, a non-invasive imaging biomarker, has the potential for use in evaluating the therapeutic response of prostate cancer. The ADC value may indicate changes in cancer at the cellular level, such as the breakdown of cancer cells or the distortion of microstructures. An increase in the ADC value reflects increased water mobility through loss of membrane integrity or an increase in the proportion of total extracellular fluid due to a decrease in cell size or number. Thus, radiotherapy-induced cellular change in prostate cancer can be non-invasively detected by DWI. 
Table III. Paired comparison of the apparent diffusion coefficient (ADC) value of tumor and healthy control tissue in the 37 patients with prostate

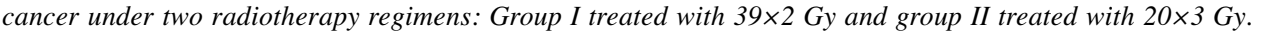

\begin{tabular}{|c|c|c|c|c|c|c|}
\hline \multirow{3}{*}{ Timepoint } & \multicolumn{6}{|c|}{$\mathrm{ADC}\left(\times 10^{-3} \mathrm{~mm}^{2} / \mathrm{s}\right)$, mean $\pm \mathrm{SD}$} \\
\hline & \multicolumn{2}{|c|}{ Group I $(\mathrm{N}=23)$} & \multicolumn{2}{|c|}{ Group II $(\mathrm{N}=20)$} & \multicolumn{2}{|c|}{ Total $(\mathrm{N}=43)$} \\
\hline & Tumor & Control & Tumor & Control & Tumor & Control \\
\hline Baseline & $0.87 \pm 0.13$ & $1.34 \pm 0.15^{\# \# \#}$ & $0.82 \pm 0.10$ & $1.42 \pm 0.14^{\# \# \#}$ & $0.84 \pm 0.12$ & $1.38 \pm 0.15^{\# \# \#}$ \\
\hline 3 Months after RT & $1.15 \pm 0.12 * * *$ & $1.34 \pm 0.14^{\# \# \#}$ & $1.25 \pm 0.12 * * *$ & $1.41 \pm 0.10^{\# \# \#}$ & $1.19 \pm 0.13^{* * *}$ & $1.38 \pm 0.13^{\# \# \#}$ \\
\hline 12 Months after RT & $1.26 \pm 0.13^{*}$ & $1.40 \pm 0.12^{\# \# \#}$ & $1.35 \pm 0.11 *$ & $1.39 \pm 0.11$ & $1.30 \pm 0.13 * * *$ & $1.39 \pm 0.11^{\# \# \#}$ \\
\hline
\end{tabular}

N: Number of lesions. RT: Radiotherapy. Significantly different at: ${ }^{*} p<0.01$ and ${ }^{* * *} p<0.0001$ compared to previous time point in the same group; $\# \# p<0.0001$ between tumor and control groups at the same time point.

Takayama et al. reported that the tumor ADC value increased significantly after carbon-ion radiotherapy in nine patients with prostate cancer (21). Song et al. demonstrated that the tumor ADC value increased significantly in both peripheral and transitional zones 1-5 months after completion of external beam radiotherapy in 49 patients with prostate cancer, whereas that of benign tissue in the peripheral and transitional zone decreased significantly after radiotherapy (13). Similarly, Part et al. reported that the tumor ADC value increased significantly during and 1 month after radiotherapy in eight patients with prostate cancer (14). Recently, Decker et al. demonstrated that the ADC value increased during, immediately and 3 months after intensity-modulated external beam radiotherapy in 13 patients with prostate cancer, but the ADC value of healthy prostate tissue did not change significantly after radiotherapy (16), which probably reflects its higher radio-resistance (22). In agreement with these studies, our results showed a significant increase of the tumor ADC value both at 3 and 12 months after radiotherapy. However, the ADC value of benign prostatic tissues did not change significantly after radiotherapy. A retrospective study of 78 patients with prostate cancer showed that the increase of tumor ADC is an indicator of effective treatment (15). Our study showed that there was a more prominent increase of the tumor ADC value in the group treated with hypofractionated radiotherapy compared to the conventional-radiotherapy group at 3 months after radiotherapy. The total increase of the tumor ADC value was similar in both groups 12 months after radiotherapy. This indicates that there was no significant difference between the hypofractionated regimen and conventional fractionation but overall treatment time using hypofractionated radiotherapy was reduced from 7.8 weeks to 4 weeks.

The $\alpha / \beta$ ratio from the linear-quadratic radiation response model used in radiation oncology is a common way of expressing the sensitivity of tumor and surrounding normal tissues to irradiation dose and fraction size (23). For most types of cancer, the $\alpha / \beta$ ratio is considered high (around $10 \mathrm{~Gy}$ ), indicating that these tissues are more sensitive to the total radiation dose than to the dose per fraction. For the latereacting surrounding normal tissues, the $\alpha / \beta$ ratio is considered low (around $3 \mathrm{~Gy}$ ), indicating a higher sensitivity to fraction dose. Emerging evidence from experimental and clinical studies suggests that the $\alpha / \beta$ ratio for prostate cancer may be as low as 1.5 to $1.8 \mathrm{~Gy}(17,18)$, which has prompted investigators to explore hypofractionated radiotherapy. Hypofractionated radiotherapy might offer both therapeutic and economic advantages over conventional radiotherapy schedules. However, long-term data about the efficacy and safety of hypofractionated radiotherapy in prostate cancer are not well established. Our patients are continuously monitored by symptom questionnaires and serum PSA; although PSA has been shown to have a limited role in defining a cancer cure within the first 5 years after radiotherapy (2). In this study, the mean PSA level had decreased significantly at 3 months after radiotherapy and decreased further 12 months after radiotherapy. However, there was one patient with PSA bounce 3 months after radiotherapy.

This study had several limitations. Firstly, we were unable to evaluate the correlation between MRIs and histopathological features accurately because we did not obtain surgical specimens. Secondly, all patients underwent needle biopsies before MRI examinations, implying that hemorrhagic or inflammatory changes caused by this procedure might have affected the MRIs. However, we excluded visible bleeding with the help of pre-contrast T1-weighted images. Finally, this study had the potential error of ADC measurements in tumors because of volume shrinkage after radiotherapy, although the ADC measurement was performed carefully by comparing the ADC map, DWI, T2-weighted images, and contrast-enhanced dynamic images.

In conclusion, the DWI ADC value is a suitable biomarker for evaluation of radiotherapy response in prostate cancer and allows for dynamic monitoring of the treatment efficacy. The moderate hypofractionation regimen is not inferior to the conventional radiotherapy regimen in the short-term observation. 


\section{Competing Interests}

The Authors have no competing interests to declare.

\section{Funding}

This project was supported by the Competitive State Research Financing of the Expert Responsibility area of Tampere University Hospital. Seppo Nieminen Fund (grant 150613) and Pirkko Kellopupu-Lehtinen (grant 9R019 and 9S021). Xingchen Wu was supported by the Emil Aaltonen Foundation and the Finnish Cultural Foundation, Pirkanmaa Regional Fund.

\section{Acknowledgements}

The Authors would like to thank research coordinator Irja Kolehmainen for her contributions.

\section{References}

1 Jereczek-Fossa BA and Orecchia R: Evidence-based radiation oncology: definitive, adjuvant and salvage radiotherapy for nonmetastatic prostate cancer. Radiother Oncol 84(2): 197-215, 2007.

2 Roach M, 3rd: Commentary on a multi-institutional analysis of external beam radiotherapy for T1-T2 prostate cancer: "love the one you're with" and "do the right thing". Int J Radiat Oncol Biol Phys 57(4): 907-909, 2003.

3 Hanlon AL, Pinover WH, Horwitz EM and Hanks GE: Patterns and fate of PSA bouncing following 3D-CRT. Int J Radiat Oncol Biol Phys 50(4): 845-849, 2001.

4 Vicini FA, Vargas C, Abner A, Kestin L, Horwitz E and Martinez A: Limitations in the use of serum prostate specific antigen levels to monitor patients after treatment for prostate cancer. J Urol 173(5): 1456-1462, 2005.

5 Boccon-Gibod L and Djavan WB, Hammerer P, Hoeltl W, Kattan MW, Prayer-Galetti T, Teillac P and Tunn UW: Management of prostate-specific antigen relapse in prostate cancer: a European Consensus. Int J Clin Pract 58(4): 382-390, 2004.

6 Stephenson AJ, Scardino PT, Bianco FJ Jr. and Eastham JA: Salvage therapy for locally recurrent prostate cancer after external beam radiotherapy. Curr Treat Options Oncol 5(5): 357-365, 2004.

7 Akin $\mathrm{O}$ and Hricak H: Imaging of prostate cancer. Radiol Clin North Am 45(1): 207-222, 2007.

8 Fuchsjager M, Akin O, Shukla-Dave A and Pucar D and Hricak $\mathrm{H}$ : The role of MRI and MRSI in diagnosis, treatment selection, and post-treatment follow-up for prostate cancer. Clin Adv Hematol Oncol 7(3): 193-202, 2009.

9 Hricak H, Choyke PL, Eberhardt SC, Leibel SA and Scardino PT: Imaging prostate cancer: a multidisciplinary perspective. Radiology 243(1): 28-53, 2007.

10 Sala E, Eberhardt SC, Akin O, Moskowitz CS, Onyebuchi CN, Kuroiwa K, Ishill N, Zelefsky MJ, Eastham JA and Hricak H: Endorectal MR imaging before salvage prostatectomy: tumor localization and staging. Radiology 238(1): 176-183, 2006.

$11 \mathrm{Wu}$ X, Reinikainen P, Vanhanen A, Kapanen M, Vierikko T, Ryymin P, Hyödynmaa $S$ and Kellokumpu-Lehtinen PL: Correlation between apparent diffusion coefficient value on diffusion-weighted MR imaging and Gleason score in prostate cancer. Diagn Interv Imaging 98(1): 63-71, 2017.
12 Hotker AM, Mazaheri Y, Aras O, Zheng J, Moskowitz CS, Gondo T, Matsumoto K, Hricak $\mathrm{H}$ and Akin O: Assessment of prostate cancer aggressiveness by use of the combination of quantitative DWI and dynamic contrast-enhanced MRI. Am J Roentgenol 206(4): 756-763, 2016.

13 Song I, Kim CK, Park BK and Park W: Assessment of response to radiotherapy for prostate cancer: value of diffusion-weighted MRI at 3 T. Am J Roentgenol 194(6): W477-482, 2010.

14 Park SY, Kim CK, Park BK, Park W, Park HC, Han DH and Kim B: Early changes in apparent diffusion coefficient from diffusion-weighted MR imaging during radiotherapy for prostate cancer. Int J Radiat Oncol Biol Phys 83(2): 749-755, 2012.

15 Liu L, Wu N, Ouyang H, Dai JR and Wang WH: Diffusionweighted MRI in early assessment of tumour response to radiotherapy in high-risk prostate cancer. Br J Radiol 87(1043): 20140359, 2014.

16 Decker G, Murtz P, Gieseke J, Traber F, Block W, Sprinkart AM, Leitzen C, Buchstab T, Lütter C, Schüller H, Schild HH and Willinek WA: Intensity-modulated radiotherapy of the prostate: dynamic ADC monitoring by DWI at 3.0 T. Radiother Oncol 113(1): 115-120, 2014.

17 Dasu A: Is the alpha/beta value for prostate tumours low enough to be safely used in clinical trials? Clinical oncology 19(5): 289301, 2007.

18 Dearnaley D, Syndikus I, Mossop H, Khoo V, Birtle A, Bloomfield D, Graham J, Kirkbride P, Logue J, Malik Z, Money-Kyrle J and O'Sullivan JM, Panades M, Parker C, Patterson H, Scrase C, Staffurth J, Stockdale A, Tremlett J, Bidmead M, Mayles H, Naismith O, South C, Gao A, Cruickshank C, Hassan S, Pugh J, Griffin C, Hall E; CHHiP Investigators: Conventional versus hypofractionated high-dose intensity-modulated radiotherapy for prostate cancer: 5-year outcomes of the randomised, non-inferiority, phase 3 CHHiP trial. Lancet Oncol 17(8): 1047-1060, 2016.

19 National Comprehensive Cancer Network Clinical Practice Guidelines in Oncology. Prostate Cancer (Version 1.2014). NCCN.org.

20 Zelhof B, Pickles M, Liney G, Gibbs P, Rodrigues G, Kraus S and Turnbull L: Correlation of diffusion-weighted magnetic resonance data with cellularity in prostate cancer. BJU Int 103(7): 883-888, 2009.

21 Takayama Y, Kishimoto R, Hanaoka S, Nonaka H, Kandatsu S, Tsuji H, Tsujii H, Ikehira $\mathrm{H}$ and Obata T: ADC value and diffusion tensor imaging of prostate cancer: changes in carbon-ion radiotherapy. J Magn Reson Imaging 27(6): 1331-1335, 2008.

22 Barnett GC, West CM, Dunning AM, Elliott RM, Coles CE, Pharoah PD and Burnet NG: Normal tissue reactions to radiotherapy: towards tailoring treatment dose by genotype. Nat Rev Cancer 9(2): 134-142, 2009.

23 Fowler JF: The linear-quadratic formula and progress in fractionated radiotherapy. Br J Radiol 62(740): 679-694, 1989.

Received July 7, 2017

Revised July 31, 2017 Accepted August 4, 2017 